\title{
Variation, selection and heritability of thermal reaction norms for juvenile growth in Orchesella cincta (Collembola: Entomobryidae)
}

\author{
GERARD DRIESSEN, JACINTHA ELLERS and Nico M. VAN STRAALEN
}

\begin{abstract}
Department of Animal Ecology, Institute of Ecological Science, Faculty of Earth and Life Sciences, Vrije Universiteit, De Boelelaan 1085, 1081 HV, Amsterdam, The Netherlands; e-mail: gerard.driessen@ecology.falw.vu.nl
\end{abstract}

\begin{abstract}
Key words. Phenotypic plasticity, slope of reaction norm, growth rate, temperature, genetic variation, heritability, Entomobryidae, Orchesella cincta
\end{abstract}

\begin{abstract}
Genetic variation for thermal plasticity plays an important role in the success or failure of a species with respect to the colonization of different thermal habitats and the ability to deal with climatic change. The aim of this paper is to study the relative contribution of the additive and non-additive components of genetic variation for the slope of the temperature reaction norm for juvenile growth rate in the springtail Orchesella cincta. We present the outcome of an artificial selection experiment for steep and flat temperature reaction norms and the results of a parent-offspring heritability experiment. There was a considerable phenotypic variation for the slope of the reaction norm. The selection experiment and the offspring to parent regression analysis, however, yielded no evidence for significant additive genetic variance. There were also no indications for maternal effects. The full-sib analysis, on the other hand, revealed a significant broad sense heritability of 0.76. An unforeseen result was that the slopes of females were steeper than those of males. This influenced the broad sense heritability of the full-sib analysis, since accidental female or male biased broods inflate the estimate of heritability. A randomization test showed that the probability level of the observed "between group" variance on the basis of the sexual differences alone was less than $10^{-5}$. From this we conclude that autosomal genetic variation played its own separate role. In conclusion, the thermal reaction norm for growth in juvenile $O$. cincta is not very much determined by the additive effects of a large number of independent genes, but more likely based on a still unknown but mainly non-additive, partially sex-related genetic mechanism, possibly including both dominance and epistatic effects. Hypotheses about the role of phenotypic plasticity in processes of local adaptation and speciation should thus be alert to such a complex genetic architecture.
\end{abstract}

\section{INTRODUCTION}

Growth and developmental rates of plants and ectothermal animals are plastic traits that are strongly affected by environmental temperature. Below a particular lower threshold temperature the metabolism of ectotherms is practically idle and no growth or development occurs. Above this threshold growth and developmental rates increase more or less linearly with temperature up to an upper limit after which further temperature increments rapidly become detrimental, Gilbert \& Raworth, 1996; Trudgill et al., 2005). The pattern is based on longestablished empirical data and described for many animal species (e.g., Bliss, 1926; Atlas, 1935; van Straalen \& Joosse, 1985; Leach et al., 1986) and many, often agricultural, plant species (e.g., Summerfield et al., 1991; Qi et al., 1999). The functional relationship between temperature and performance is called a thermal reaction norm. A roughly linear relationship in the increasing part of the temperature reaction norm is also rather common for many behavioural and physiological traits (Angilletta et al., 2002).

Thermal reaction norms can differ between species, between populations, between individuals, between sexes and between different developmental stages within an individual (van Straalen, 1994; Stam, 1997; Li, 1998; Jarošík et al., 2002), and they can vary in several ways (Huey \& Kingsolver, 1989; van der Have \& de Jong,
1996; Kingsolver et al., 2004). They may, for example, be of similar shape but with different temperatures of maximum performance, i.e., shifted horizontally along the temperature axis (e.g., the ovariole number in the fruit fly Drosophila ananassae, Yadav \& Singh, 2005). They can also be of similar shape but shifted vertically along the performance axis, implying that some individuals perform faster or bigger at all temperatures (e.g., egg size in the tropical butterfly Bicyclus anynana, Steigenga et al., 2005). Finally, reaction norms can also differ in shape, for example steep reaction norms over a relatively narrow temperature interval with a high maximum performance versus flat reaction norms over a broad temperature range with lower maximum performance (e.g., body size in Drosophila melanogaster, David et al., 2006). It is noteworthy to keep in mind that, in terms of plasticity, the flat type is the least plastic, since its performance is least influenced by temperature.

The nature of the genetic mechanism that determines the position of a thermal reaction norm can have important consequences for evolutionary processes. If growth or development rates at different temperatures are genetically correlated, then the reaction norm itself can be considered as a trait "as a whole" that is potentially subject to selection. As a consequence growth or developmental rates at different temperatures cannot evolve independently. Clearly, this would have important consequences 
for the success or failure of a species with respect to the colonization of different thermal habitats or adaptation to climatic change.

In Collembola (springtails) several studies have measured temperature reaction norms for a variety of life-history traits: egg size, egg development rate, fecundity, moulting rate, age at maturity and others (e.g., van Straalen \& Joosse, 1985; Stam, 1997). Van Straalen (1994) found a considerable variation in the slope of the thermal reaction norm for egg development rate in a group of 34 collembolan species. In the present study we are primarily interested in the genetic nature of the variation for steep (most plastic) versus flat (least plastic) thermal reaction norms for juvenile growth rate at the within species level in the collembolan Orchesella cincta L.

Juvenile growth rate is an important life-history character especially for ectotherms that live in a seasonal environment, since it can play a role in the synchronization of the reproduction in a population. Additionally, growth rate affects the degree of exposure to predation during the more vulnerable juvenile stages. If the thermal reaction norm is "a trait as a whole" and the genetic variance is mainly additive it must be possible to select for steep and flat reaction norm slopes. Here, we will present the outcome of such an artificial selection in $O$. cincta and the results of a parent-offspring heritability experiment intended to reveal the relative contribution of the additive and non-additive components of genetic variation for slope.

\section{MATERIAL AND METHODS}

\section{Animals and experimental populations}

Orchesella cincta $\mathrm{L}$. is a surface-dwelling collembolan (adult size 3-4 mm). It occurs over large part of Europe and Asia and around the Mediterranean and can be found in very different types of thermal habitats, from fairly temperature stable dense pine forests to highly exposed moors and coastal dune areas, where it lives in small isolated populations. In the Netherlands $O$. cincta has two generations a year. Juveniles pass through around 11 non-reproductive moults before they are mature. In the adult stage reproductive and non-reproductive periods alternate separated by moults. In a reproductive period female O. cincta lay their eggs after taking up one single spermatophore deposited on the substrate by a male (Gols et al., 2004).

For the selection experiment animals were sampled from four natural populations, one in Poland (Pilica: $50^{\circ} 29.1^{\prime} \mathrm{N}$, $19^{\circ} 39.6^{\prime} \mathrm{E}$ ), one in Sweden (Ringarum: $58^{\circ} 21.5^{\prime} \mathrm{N}, 16^{\circ} 13.7^{\prime} \mathrm{E}$ ) and two in the Netherlands (Roggebotzand: $52^{\circ} 34.4^{\prime} \mathrm{N}$, $05^{\circ} 47.9^{\prime} \mathrm{E}$ and Amsterdamse Waterleidingduinen: $52^{\circ} 1.5^{\prime} \mathrm{N}$, $\left.04^{\circ} 33.1^{\prime} \mathrm{E}\right)$. This was done in order to increase the level of genetic variation and motivated by the fact that Stam (1997) reported a significant phenotypic variation between Italian, Norwegian and Dutch populations for egg development, moulting and maturation rate. Of each of the four sampled populations 20 individuals from the first laboratory generation produced by the field-collected animals were mixed and allowed to reproduce for two generations. This was repeated two times in order to create three replicate selection experiments (I, II and III). The offspring of the second generation is referred to as the initial F0 generation in the selection experiment. In a preliminary experi- ment that served to check the intercrossing capacity of the populations, all possible combinations of mating pairs readily produced offspring. The heritability experiment was carried out seven months after the start of the selection experiment with animals from replicate population II. By that time the original populations had been mixed for at least five generations, under rearing conditions of $20^{\circ} \mathrm{C}, \mathrm{RH}=80 \%$ and $12 \mathrm{~L}: 12 \mathrm{D}$.

\section{Measurement of the slope of the thermal reaction norm for} growth rate

A simple two-parameter linear model is often used to describe the increasing part of reaction norms for growth and development. This application of a linear approximation to thermal reaction norms has been considerably successful in predicting developmental times in numerous plants and animals (Atkinson, 1996). The slope of the reaction norm for growth was therefore defined as $\left(r_{12^{\circ} \mathrm{C}}-r_{22^{\circ} \mathrm{C}}\right) / 10$, where $r_{12{ }^{\circ} \mathrm{C}}$ and $r_{22^{\circ} \mathrm{C}}$ denote the exponential growth rate parameters at $12^{\circ} \mathrm{C}$ and $22^{\circ} \mathrm{C}$, respectively. In order to measure $r_{12^{\circ} \mathrm{C}}$ and $r_{22^{\circ} \mathrm{C}}$, freshly emerged juvenile springtails $\left(\sim 5\right.$ days old at $\left.20^{\circ} \mathrm{C}\right)$ were weighed on a microbalance (Mettler Toledo UMT2, precision $1 \mu \mathrm{g}$ ). After weighing they were stored individually in small plastic vials $(2 \mathrm{~cm}$ in diameter, $3 \mathrm{~cm}$ high) with a bottom of plaster of Paris and incubated at $12^{\circ} \mathrm{C}(\mathrm{RH}=80 \%, 12 \mathrm{~L}: 12 \mathrm{D})$. Small pieces of bark overgrown with green algae (Desmococcus sp.) served as food. Food availability was regularly checked and always kept in excess. After two weeks of incubation at $12^{\circ} \mathrm{C}$ the animals were weighed for a second time and subsequently transferred to $22^{\circ} \mathrm{C}$ $(\mathrm{RH}=80 \%, 12 \mathrm{~L}: 12 \mathrm{D})$ for another week. They were then weighed for a third time. Acclimation of growth rate to temperature change is practically immediate in $O$. cincta; growth rates during the first day after a temperature change are the same as on later days (G. Driessen, unpubl. data). After the third weight measurement the springtails were stored at $20^{\circ} \mathrm{C}$ until maturity. A two-week exposure to $12^{\circ} \mathrm{C}$ and a one-week exposure to $22^{\circ} \mathrm{C}$ was chosen because we expected a roughly similar number of moults and mean proportional weight increase using these periods.

With the three weight measurements the growth rates of each individual juvenile springtail could be calculated at both $12^{\circ} \mathrm{C}$ and $22^{\circ} \mathrm{C}$. Growth of juvenile $O$. cincta is nearly exponential up to around $600 \mu \mathrm{g}$ (Janssen \& Joosse, 1987). Therefore, we defined the juvenile growth rate $r$ at temperature $T$ as:

$$
r_{T}=\frac{1}{\Delta t} \ln \frac{w_{i+1}}{w_{i}},(i=1,2),
$$

where $w_{i}$ is the fresh weight (in $\mu \mathrm{g}$ ) at the $i^{\text {th }}$ measurement, and $\Delta t$ is the time (in days $\times 10^{-1}$ ) between the two successive weightings. The reason for choosing $12^{\circ} \mathrm{C}$ and $22^{\circ} \mathrm{C}$ was that at $8-10^{\circ} \mathrm{C}$ juvenile growth is almost zero (J. Ellers, unpubl. data), while $25^{\circ} \mathrm{C}$ is just above the temperature of maximum growth (G. Driessen, unpubl. data) and maximum egg development rate (van Straalen, 1994). Only juveniles $(<\sim 600 \mu \mathrm{g})$ were used for growth measurements.

\section{Selection experiment}

The following selection procedure was carried out. To begin with, 140 freshly emerged juveniles $(\sim 50 \mu \mathrm{g})$ were randomly selected from each of the three founding replicate populations and their growth rates at $12^{\circ} \mathrm{C}$ and $22^{\circ} \mathrm{C}$ were measured according to the procedure described above. Of these animals 78,69 and 89 individuals survived respectively until adulthood, and of each group those 20 springtails with the steepest and those 20 with the flattest slopes were allowed to reproduce. From the point of view of risk spreading, this was done in two subgroups of 10 individuals that were incubated together at $20^{\circ} \mathrm{C}(\mathrm{RH}=80 \%, 12 \mathrm{~L}: 12 \mathrm{D})$ in one vial $(5 \mathrm{~cm}$ in diameter, 3 $\mathrm{cm}$ high, with a bottom of plaster of Paris and algae as a food 
source). Because sexing of individuals is time consuming and inducing an extra mortality risk due to handling, the animals were not sexed.

This first selection round resulted in a "steep" and a "flat" line within each replicate experiment. Each subsequent generation between 70-90 juvenile springtails were measured in each of the three "steep" and "flat" lines. In the steep lines the 20 steepest and in the flat lines the 20 flattest animals were selected to produce the next generation (again in two groups of 10 animals). In total four selection rounds were carried out.

\section{Heritability of the slope of the reaction norm}

Freshly hatched juveniles $(\sim 50 \mu \mathrm{g})$ were randomly selected from the lab culture of founding population II and their growth rates were measured at $12^{\circ} \mathrm{C}$ and $22^{\circ} \mathrm{C}$ (see above). This experiment started seven months after the start of the artificial selection, which implies that the had been mixed for al least five generations since they were collected in the field. For logistic reasons the measurements were done in two separate and subsequent series (A and B) each starting with 150 animals. After the animals had developed to adulthood they were sexed. Males and females were randomly paired, incubated in small vials and checked regularly for eggs and juveniles. Due to mortality, loss or deficiency to reproduce 27 and 25 parent pairs were obtained ultimately, for which we had growth measurements at $12^{\circ} \mathrm{C}$ and $22^{\circ} \mathrm{C}$ and which reproduced enough to measure the growth rates of offspring.

The $r_{T}$-values for $O$. cincta turned out to be somewhat positively related to the initial weight. This is probably due to the fact that growth is not strictly isometric, as is also found for many other insects (Kingsolver et al., 2004). To allow pooling of the results of series A and B we transformed the $r_{T}$-values in the following way. For each series A and B separately, the growth rates at each temperature (calculated with eqn 1) were regressed on the first weight measurement for that temperature. The standardized residuals of these regressions where then pooled per temperature to produce the data set for the analysis. Therefore the mean growth rate at a particular temperature is zero, while the growth rates themselves are expressed in units of standard deviation. Growth rates of five offspring of each pair were obtained at $12^{\circ} \mathrm{C}$ and $22^{\circ} \mathrm{C}$ by the same procedure and transformation as for the parents (see above). All transformed data fitted well to a normal distribution (all $P>0.20$, Kolmogorov-Smirnov one sample test). The slope of the temperature reaction norm of an individual was calculated from the difference between the transformed growth rates at $12^{\circ} \mathrm{C}$ and $22^{\circ} \mathrm{C}$ divided by 10 .

Not all offspring survived until the third weighing and occasionally the mean offspring value was based on less than 5 animals. Still, on average 4.46 offspring per pair were measured and therefore no correction for unequal family size was considered to be needed. The confidence intervals of the heritabilities were calculated according to Falconer (1989), using an REML procedure to estimate intra class correlation.

\section{RESULTS}

\section{Artificial selection}

Fig. 1 shows the reaction norms for growth rate of the individuals in the three initial populations from which the artificial selection was started. The frequency distributions of the slopes did not differ significantly from a normal distribution (minimum $P>0.20$, KolmogorovSmirnov one-sample test) and not from each other (minimum $P>0.10$, Kolmogorov-Smirnov two-sample test). There was a considerable phenotypic variation in

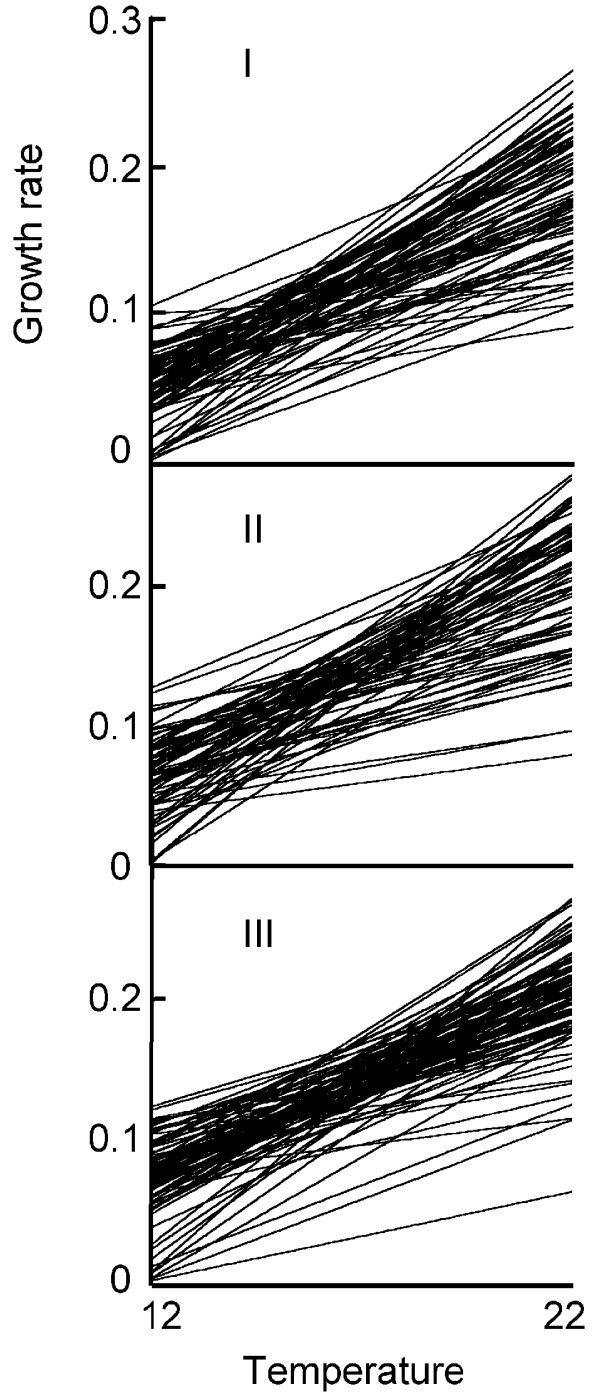

Fig. 1. Slopes of the thermal reaction norms for juvenile growth rate in $O$. cincta in the three replicate founding (F0) populations (I, II and III) of the artificial selection experiment.

slopes and the highest classes were 6 to 10 times steeper than the lowest classes (Fig. 1). Means of the slopes were $0.0131,0.0126$ and 0.0121 for population I, II and III respectively with standard deviations of $0.0047,0.0054$ and 0.0042 .

In each generation the 20 springtails with the steepest and the 20 animals with the flattest reaction norms were selected to generate the next generation. The average selection differential (defined as the percentage difference between the mean slope of the group that was selected to establish the next generation and the population mean) over all selection rounds was $43.4 \%$ (s.d. $4.7 \%$ ) in the steep selection lines and $39.1 \%$ (s.d. $11.1 \%$ ) in the flat selection lines.

Fig. 2 shows the means, standard errors and 95\% confidence intervals of the means in the four successive generations of the artificial selection experiment for each of the three replicates. The changes in the first generation were considerable; in all three replicates the slope values decreased dramatically. Moreover, the responses to selec- 


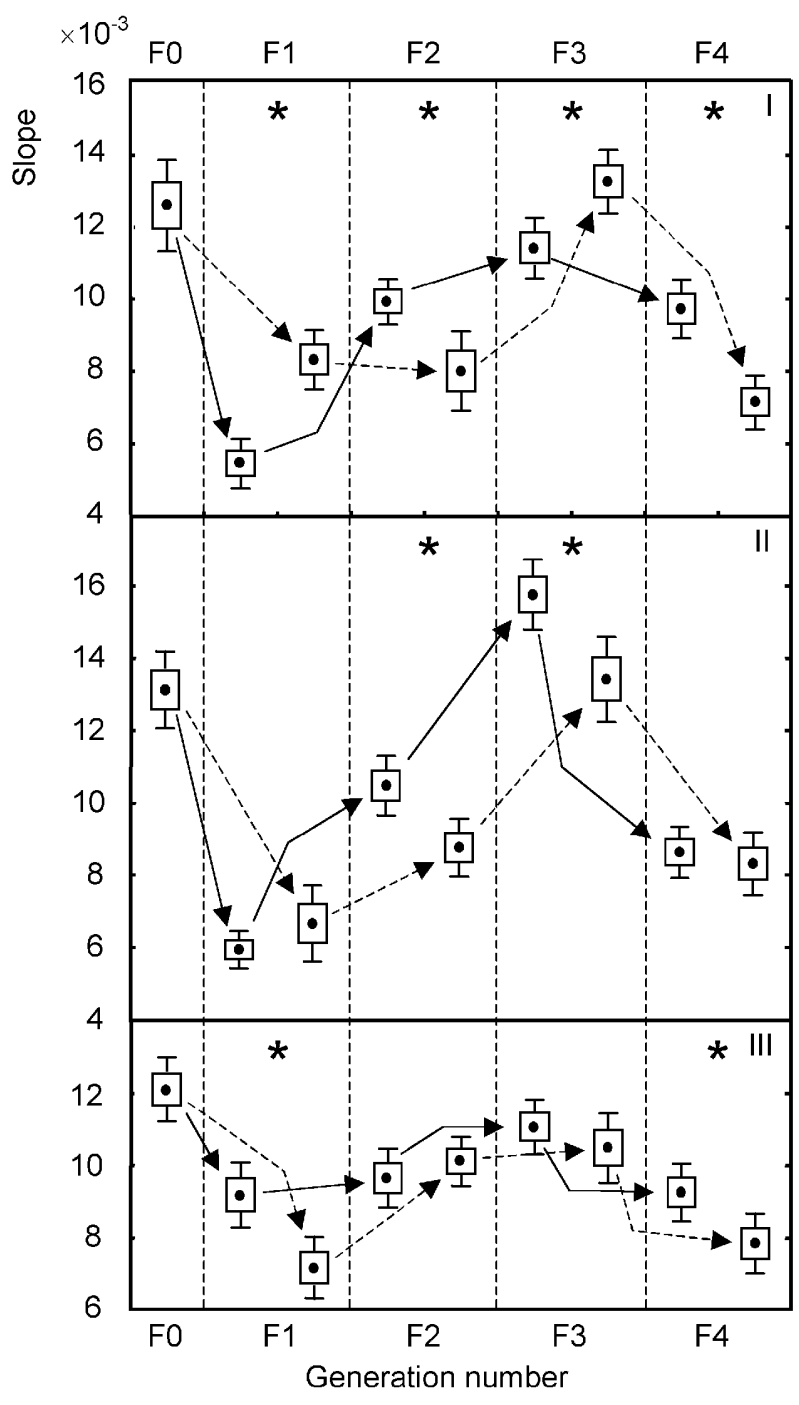

Fig. 2. Means (dots), standard errors of the means (boxes) and $95 \%$ confidence intervals of the means (whiskers) of the slopes of the thermal reaction norms for growth in $O$. cincta in successive generations (F0 to F4) in the replicates, I, II and III, of the artificial selection experiment. Solid arrows connect steep selection lines, interrupted arrows connect flat selection lines. An asterisk indicates significant differences between steep and flat populations.

tion were not consistent. Selection for steep slopes (solid arrows) should lead to relative higher population means compared to the flat lines, whereas the reverse would be expected in the selection for flat reaction norms (dashed arrows). In fact this pattern was only found in replicate III. The reverse pattern was found in replicate I and no difference in replicate II (significant differences between flat and steep lines within each generation and replicate are indicated by an asterix in Fig. 2).

In the second generation and third generation the overall value of the slopes gradually increased to levels comparable to the initial F0 population. As for the differences between the flat and steep lines the patterns were as expected in replicate I and II in the F2 generation, but differences disappeared in replicate III. In the F3 generation the order of the lines in replicate I reversed and remained

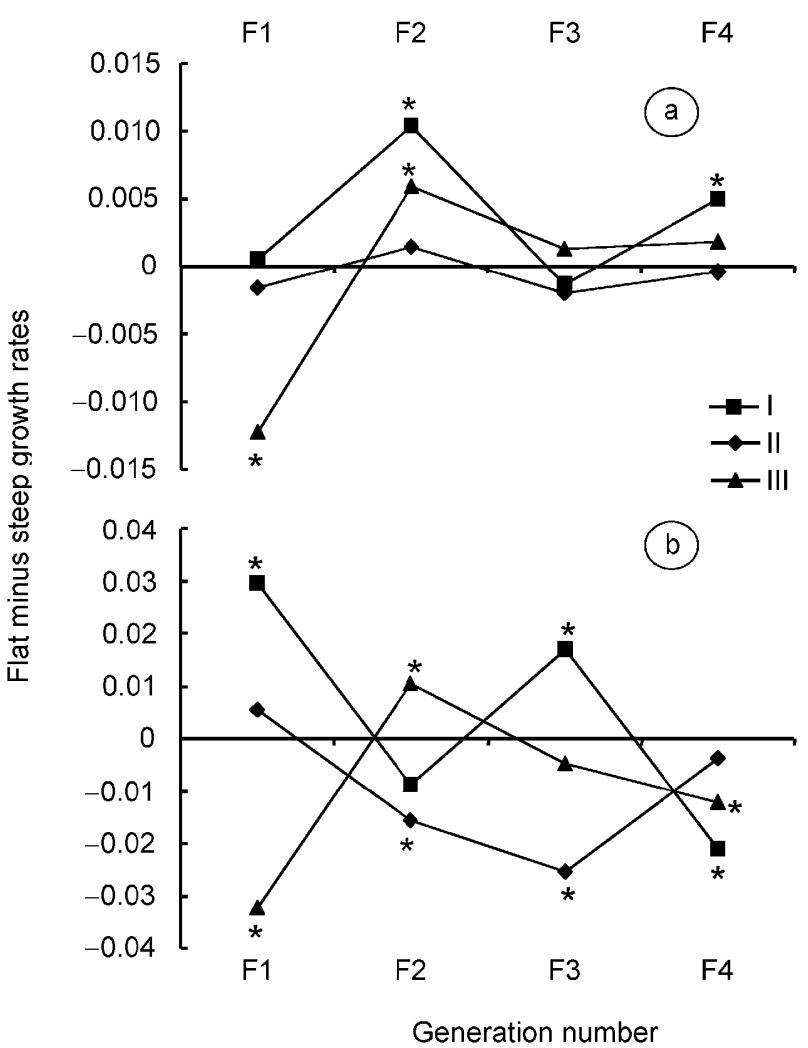

Fig. 3. The mean growth rates of the flat lines minus the mean growth rates of the steep lines in the replicates, I, II and III, of selection experiment at the two temperatures $12^{\circ} \mathrm{C} \mathrm{(a)} \mathrm{and} 22^{\circ} \mathrm{C}$ (b). Means significantly different from zero are indicated by an asterisk.

the same in replicate II. In replicate III differences remained not significant.

In the fourth generation the overall value of the slopes decreased again in all replicates. In replicate I the order of the selection lines reversed again into the expected direction, and in replicate III, differences became significant in the expected direction. In replicate II the differences disappeared.

All in all, the mutual dynamics of the selection for steep and flat lines were rather different between replicates: flat and steep selection lines irregularly swapped relative position and apparently stable differences sometimes suddenly disappeared. After four generations there were significant differences in reaction norm slopes in the expected direction in two out of the three replicates (I and III), but given the erratic patterns in the preceding generations no firm conclusions can be drawn.

The differences in the slopes in I and III by the fourth generation could have come up in two different ways: one is through a negative correlation between the growth rates at $12^{\circ} \mathrm{C}$ and $22^{\circ} \mathrm{C}$, or secondly through an increase or decrease of the growth rate at one temperature in combination with no change at the other. Fig. 3 gives the answer to this question. In this figure the mean growth rates of the flat lines minus the mean growth rates of the steep lines at the two temperatures $12^{\circ} \mathrm{C}$ (Fig. 3a) and $22^{\circ} \mathrm{C}$ (Fig. 3b) are plotted. If the differences in slopes are 


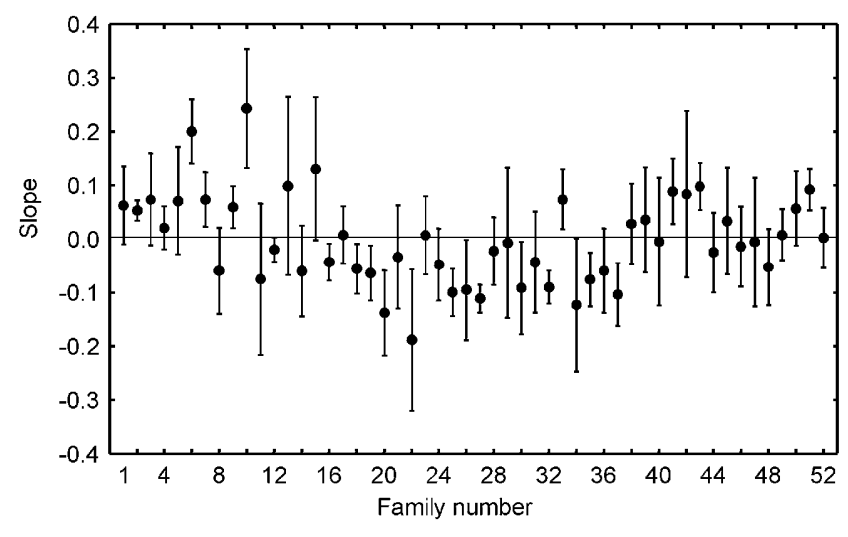

Fig. 4. Means (dots) and 95\% confidence intervals of the means (whiskers) of the slopes of the thermal reaction norms for juvenile growth in $O$. cincta of each family of full-sibs in the offspring generation of the heritability experiment. Note that the slopes are based on standardized growth rates; negative values correspond to flat slopes, positive values to steep slopes.

caused by a negative correlation between growth rates then the points are expected to gradually move up to above the $\mathrm{X}$-axis in Fig. 3a, and to move down to below the $\mathrm{X}$-axis in Fig. 3b. We see indeed that by the fourth generation there was a general trend in this direction. More specifically, in replicate I, the steep line grew significantly slower at $12^{\circ} \mathrm{C}(P=0.009, t$-test, $n=92 / 84$, Fig. 3a) and significantly faster at $22^{\circ} \mathrm{C}\left(P=10^{-4}\right.$, $t$-test, $n$ $=92 / 84$, Fig. 3b). Similar results were found in replicate III but significance was only detected at $22^{\circ} \mathrm{C}(P=0.046$, $t$-test, $n=85 / 91$, Fig. 3b).

\section{Heritability experiment}

The broad sense heritability estimate, $h^{2}$, of the slope of the thermal reaction norm according to the full-sib analysis was equal to 0.76 and significantly different from zero $(95 \%$ confidence interval not including zero, Table 1). This is illustrated in Fig. 4 where a large among family variation in slope can be observed. Note that the slopes are based on standardized growth rates, which means that negative values in Fig. 4 correspond to flat (but not necessarily negative) thermal reaction norm slopes and positive values to steep slopes.

Fig. 5a shows the mean offspring on midparent regression of the slopes in the heritability experiment. The narrow sense heritability that could be estimated from this regression was equal -0.082 and was not statistically different from zero (Table 1). Hence, parents were bad predictors for their offspring with respect to the slope of the thermal reaction norm. Similar results appeared when offspring was regressed on mothers (Fig. 5b), or fathers
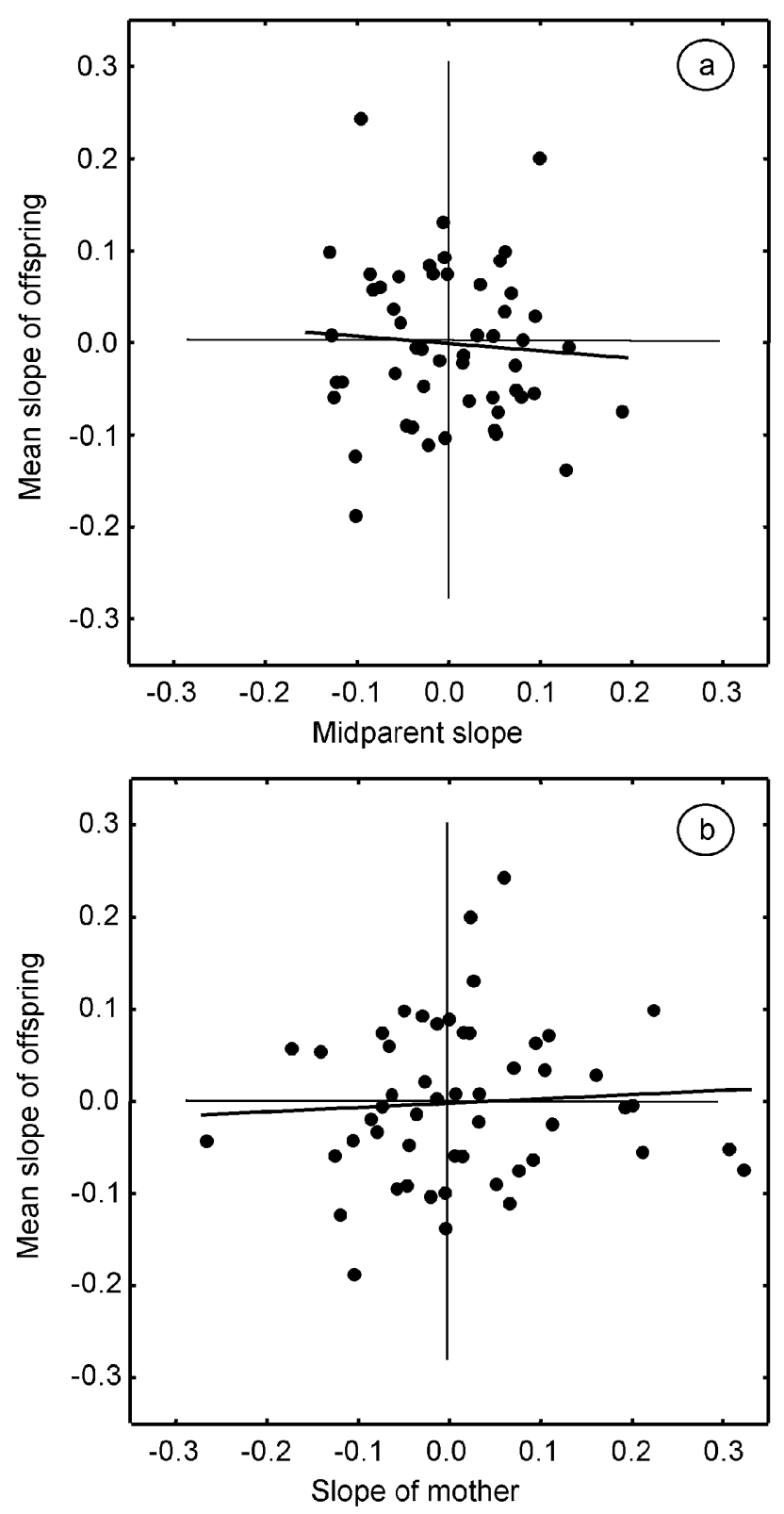

Fig. 5. Mean offspring to midparent regression (a) and mean offspring to mother regression (b) for the standardized slopes of the thermal reaction norms for juvenile growth in $O$. cincta in the heritability experiment.

separately (Table 1), indicating that there were no maternal or paternal effects.

An unforeseen result revealed by the parent population in heritability experiment was a sex-related component in reaction norm slope. At $12^{\circ} \mathrm{C}$ the growth rates of mothers and fathers were not significantly different $(P=0.227$,

TABLE 1. Heritability, $h^{2}$, of the slope of the thermal reaction norm for growth, standard errors, and $95 \%$ confidence intervals, determined by different methods (for calculations see Falconer, 1989, $n=4.46, N=52, t$ based on REML, no correction for unbalanced design).

\begin{tabular}{lccc}
\hline Method & $h^{2}$ & s.e. $\left(h^{2}\right)$ & $95 \%$ confidence interval of $h^{2}$ \\
\hline Full-sib analysis & 0.761 & 0.284 & $0.477<h^{2}<1.044$ \\
Offspring on midparent & -0.082 & 0.142 & $-0.360<h^{2}<0.195$ \\
Offspring on mother & 0.097 & 0.200 & $-0.296<h^{2}<0.489$ \\
Offspring on father & 0.199 & 0.200 & $-0.193<h^{2}<0.591$ \\
\hline
\end{tabular}


$t$-test, $n=52$ ). However, at $22^{\circ} \mathrm{C}$ the "raw" (not standardized) growth rates, $r$, of the females were around $10 \%$ higher than those of the males $(P=0.009, t$-test, $n=52$, test on pooled transformed data). These two findings make that females had steeper slopes than males, and also higher overall growth rates $(P=0.026$ and $P=0.007$ respectively). This causes a problem for the full-sib analysis above. The fact that some families of sibs have steeper or flatter slopes than others (Fig. 4) may thus partly have been caused by accidental sex ratio differences between these families. The maximum family sample size was 5; hence the occurrence of all male or all female broods was not very unlikely.

Unfortunately, the offspring in the heritability experiment was not sexed. Therefore a randomization procedure was applied in order to estimate the probability of finding the observed ratio of "between group" to "total" variance if only accidental sex ratio bias had been the cause of the heritability (i.e. between group variation). In each simulation run 52 families were created (of the same size as in the experiment) with a random number of male and female sibs (sex ratio is $50 \%$ in $O$. cincta). It was assumed that the difference in slope between male and female sibs was of the same order of magnitude as in the parent population. This is justified because all the measurements were standardized residuals in both parent and offspring populations. Next, slopes from the mother population were randomly assigned (with replacement) to each female offspring, and slopes from the father population to each male offspring. After each of the 52 families had been formed this way, the ratio of the "between group" to the "total" variation was calculated for that run. The ratio is thus based on the effect of sex only, while all other possible genetic effects are randomized. After 100,000 runs there was not one ratio greater or equal than the observed one. Hence, the probability level of finding the observed heritability as a result of only the difference between the sexes was less than $10^{-5}$. From this we conclude that other, autosomal genetic effects must have attributed too to the significance level of heritability. The between group variation in the simulations amounted on average $22.4 \%$ of the total variation, whereas this was $50.9 \%$ in the observed families. This implies that around $45 \%$ of the observed among family variation in the above full-sib analysis is explained by the sexual composition of the families.

\section{DISCUSSION}

With respect to measuring phenotypically plastic traits in insects it is often not possible to quantify the trait values in the different environments on the same individual. This study is an exception to this "rule", although it was not feasible to measure in more than two environments. We found a considerable phenotypic variation for the slope of the thermal reaction norm for growth rate in juvenile Orchesella cincta. Total phenotypic variance results from genetic factors (additive genetic variation, dominance effects and epistasis), maternal effects and environmentally induced variation (Falconer, 1989; Roff,
2002). The most important environmental factors for growth and development in Collembola are temperature, humidity and food. We have carefully tried to keep these factors constant and we found no indications in our computerized temperature and humidity registrations that any of these factors varied in such a way that it could have explained our results. All animals were raised individually and their positions in the climate rooms were regularly changed. Food was never observed to have been a limiting factor. The offspring to mother regression suggested no indications for maternal effects. A negative maternal effect on age at first reproduction in $O$. cincta has been reported by Janssen et al. (1988). However, this result could not be confirmed in a later study by Stam et al. (1998). Therefore, we conclude that the observed differences in plasticity must have mainly resulted from genetic variance. If the genetic contribution to the reaction norm variability in this study had been mostly additive, we should have found a gradually increasing difference between the steep (plastic) and flat (less-plastic) selection lines in the selection experiment and a significant and positive heritability for slope in the offspring to midparent regression. Although the selection differentials were substantial in the artificial selection experiment, the response to selection was not very consistent and only by the fourth generation a weak divergence between lines could be detected. Furthermore, the estimate of the heritability of the slope of the reaction norm was negative and not significantly different from zero. Thus far, these results do not support a straightforward additive genetic mechanism in juvenile $O$. cincta.

It could be argued that the erratic patterns in the selection experiment result from a relatively large linkage disequilibrium in the initial population that had not yet been able to decay. From this point of view it would have been better to start the selection experiment after three to four generations in the lab instead of two. However, the heritability experiment was carried out with animals that had been mixed for at least five generations and although linkage disequilibrium in this population had thus greatly been able to brake up, there was no evidence for additive genetic variability in this experiment either. In contrast, in the absence of a parent-offspring correlation, the significant heritability in the full-sib analysis suggests a mostly non-additive genetic component to temperature plasticity, even after controlling for the inflating effects of sexrelated differences.

The following considerations may give some further support to the existence of a complex, non-additive genetic architecture. First, there is a remarkable synchrony in the overall variation of the reaction norms in the successive generations of the selection experiment (Fig. 2). Given the magnitude of the variation it is difficult to imagine that some common, unnoticed environmental factor has been the cause of it. Moreover, for logistic reasons the measurements were commonly spread over periods of 5 to 10 days. The synchrony therefore might be the result of some intrinsic feature of the genetic mechanism. Second, the finding in this study that females 
and males have different growth rates at $22^{\circ} \mathrm{C}$, and different reaction norms slopes indicates a partially sexrelated basis. Orchesella cincta, like many other Collembola, has $\mathrm{XX} / \mathrm{XO}$ sex determination, where $\mathrm{XO}$ determines the male sex. Third, the fact that 8 out of the 12 steep/flat comparisons in the F1 to F4 generations of the selection experiment were significant (albeit in different directions) can hardly be attributed to stochastic variation only (Fig. 2). The probability of 8 type I errors out of 12 tests is $<10^{-10}$.

All in all, the slope of the thermal reaction norm for juvenile growth rate in Orchesella cincta does not seem to have a strong additive component caused by a large number of independent genes, but is more likely based on a still unknown but mainly non-additive, partially sexrelated genetic mechanism, possibly including both dominance and epistatic effects. In a review on artificial selection experiments on plastic traits, Scheiner (2002) concluded that although plasticity can be a heritable and evolvable trait, the genetics generally create complex evolutionary dynamics. The results of this study are clearly in line with this conclusion.

Life-history traits frequently show lower heritabilities than morphological, behavioural and physiological traits (Mousseau \& Roff, 1987) and generally have relatively large non-additive variance components (Roff, 1997). Many studies have reported on genetic variation in lifehistory traits of insects in relation to temperature. Traits that often have been studied in this respect are developmental rate, diapause, survival, age at maturity, adult size and fecundity. Genetic variation for growth rate, however, has received relatively little attention. This is all the more surprising since growth rate and its dependence on temperature is an important life-history variable especially for organisms that live in seasonal environments (Abrams et al., 1996). Simons et al. (1998) found genetic variation for juvenile growth rate in the cricket Gryllus pennsylvanicus when rearing offspring of field-caught individuals. Similarly, Ballabeni \& Rahier (2000) found genetic variation for growth rate in the leaf beetle Oreina elongata and Davidowitz et al. (2004) in the tobacco hornworm Manduca sexta. Kingsolver et al. (2004) estimated broad-sense heritabilities for thermal sensitivity of growth rate of caterpillars of the cabbage white butterfly Pieris rapae. They measured growth rates over a range of temperatures and found that genetic variance for growth rate increased with temperature. They also found a negative correlation between growth rates at $35^{\circ} \mathrm{C}$ and $40^{\circ} \mathrm{C}$ suggesting a genetic trade-off at these temperatures. Unfortunately, none of these studies included a parent offspring experiment, so it is difficult to separate the additive from the non-additive components of the genetic variance.

To determine whether the thermal plasticity for growth rate in $O$. cincta is a "trait as a whole", or the result of different genes operating at different temperature ranges, the best method would be to split strains in a number of lines and subsequently rear the lines at different temperatures. If, after a number of generations, the performance of these lines at temperatures other than their own rearing temperature has changed, that would be good evidence for the "a trait as a whole" hypothesis. However, the broad sense heritability found in this study can also be interpreted as supportive for this hypothesis.

A general problem when studying growth rates is that multiple measurements over time on the same individual are unavoidable. This could create a problem since errors in $w_{i}$ can potentially have a large influence on estimates of $r_{T}$ (see eqn 1). Nevertheless, we have several arguments to have good faith in our measurements. First, the impact of the influence strongly depends on the ratio $w_{i+1} / w_{\mathrm{i}}$. Especially when this ratio is large, errors in $w_{i}$ will have a large impact. In our experiments, however, the $w_{i+1} / \mathrm{w}_{i}$ ratio varied between 2 and 4 . Second and most importantly, the measurements did detect the relatively small differences between female and male growth rates in the two independent parts of the heritability experiment. Therefore, we believe that the measurements of $w_{i}$ were sufficiently accurate.

In conclusion, the non-additive nature of the genetics underlying the thermal reaction norm for growth of juvenile $O$. cincta was most probably the cause of the variable responses to selection and only by the fourth generation in the selection experiment some differentiation might have started to come to light. Adaptation to a different thermal environment by $O$. cincta under natural selection may therefore be limited by the genetic architecture and may take many generations. The effect of temperature change on the performance of organisms receives a lot of attention these days. The results of this study underline that hypotheses about the role of phenotypic (in casu growth rate) plasticity in processes of local adaptation and speciation should be alert to the fact that the genetic background of plastic traits may have a considerable nonadditive and sex-related nature.

ACKNOWLEDGEMENTS. GD was supported by the Netherlands Organization for Scientific Research, grant ALW-812.04.002 and JE was supported by the Netherlands Organization for Scientific Research, VIDI-grant 864.03.003.

\section{REFERENCES}

Abrams P.A., Leimar O., Nylin S. \& Wiklund C. 1996: The effect of flexible growth rates on optimal sizes and development times in a seasonal environment. Am. Nat. 147: 381-395.

Angilletta M.J., Niewiarowski P.H. \& Navas C. 2002: The evolution of thermal physiology in ectotherms. J. Therm. Biol. 27: 249-268.

ATKINSON D. 1996: Ectotherm life-history responses to developmental temperature. In Johnson A. \& Bennett A.F. (eds): Animals and Temperature. Phenotypic and Evolutionary Adaptation. Cambridge University Press, Cambridge, pp. 183-204.

AtLAS M. 1935: The effects of temperature on the development of Rana pipiens. Physiol. Zool. 8: 290-310.

BALlabeni P. \& RAHIER M. 2000: A quantitative genetic analysis of leaf beetle larval performance on two natural hosts: including a mixed diet. J. Evol. Biol. 13: 98-106. 
BLISS C.I. 1926: Temperature characteristics for prepupal development of Drosophila melanogaster. J. Gen. Physiol. 9: 476-495.

David J.R., Legout H. \& Moreteau B. 2006: Phenotypic plasticity of body size in a temperate population of Drosophila melanogaster: when the temperature-size rule does not apply. J. Genet. 85: 9-23.

Davidowitz G., D’Amico L.J. \& Nishout H.F. 2004: The effects of environmental variation on a mechanism that controls insect body size. Evol. Ecol. Res. 6: 49-62.

FALCONER D.S. 1989: Introduction to Quantitative Genetics. Longmans, New York, 438 pp.

Gilbert N. \& Raworth D.A. 1996: Insects and temperature - a general theory. Can. Entomol. 128: 1-13.

Gols R., ERnsting G. \& van Straalen N.M. 2004: Paternity analysis in a hexapod (Orchesella cincta; Collembola) with indirect sperm transfer. J. Insect Behav. 17: 317-328.

Huey R.B. \& Kingsolver J.G. 1989: Evolution of thermal sensitivity of ectotherm performance. TREE 4: 131-135.

JANSSEN G.M. \& Joosse E.N.G. 1987: Reproduction and growth in Collembola under laboratory conditions. Pedobiologia 30: $1-8$.

Janssen G.M., de Jong G., Joosse E.N.G. \& Scharloo W. 1988: A negative maternal effect in springtails. Evolution 42: $828-834$.

JARošíK V., HoNĚK A. \& Dixon A.F.G. 2002: Developmental rate isomorphy in insects and mites. Am. Nat. 160: 407-510.

Kingsolver J.G., Ragland G.J. \& Shlichta J.G. 2004: Quantitative genetics of continuous reaction norms: thermal sensitivity of caterpillar growth rates. Evolution 58: 1521-1529.

Leach L., Trudgill D.L. \& Gahan P.B. 1986: Influence of food and temperature on the development and moulting of Goodeyus ulmi (Nematoda: Rhabditida). Nematologica 32: 216-221.

Li D. 1998: A linear model for description of the relationship between the lower threshold temperature and thermal constant in spiders (Araneae: Arachnida). J. Therm. Biol. 23: 23-30.

MousSEAU T.A. \& RoFF D.A. 1987: Natural selection and the heritability of fitness components. Heredity 59: 181-198.

Qi A., Wheeler T.R., Keatinge J.D.H., Ellis R.H., SummerFIELD R.J. \& CRAUfURD P.Q. 1999: Modelling the effects of temperature on the rates of seedling emergence and leaf appearance in legume cover crops. Exp. Agr. 35: 327-344.

RofF D.A. 1997: Evolutionary Quantitative Genetics. Chapman and Hall, New York, $535 \mathrm{pp}$.

RoFF D.A. 2002: Life History Evolution. Sinauer Associates, Inc., Sunderland, $527 \mathrm{pp}$.

SCHEINER S.M. 2002: Selection experiments and the study of phenotypic plasticity. J. Evol. Biol. 15: 889-898.

Simons A.M., Carrière Y. \& Roff D.A. 1998: The quantitative genetics of growth in a field cricket. J. Evol. Biol. 11: 721-733.

StAM E. 1997: Temperature responses and physiological time: A comparison of three European populations of Orchesella cincta. Pedobiologia 41: 88-93.

Stam E., IsaAks A. \& Ernsting G. 1998: Negative maternal effect revisited: a test on two populations of Orchesella cincta L. (Collembola: Entomobryidae). Evolution 52: 1839-1843.

Steigenga M.J., ZwaAn B.J., Brakefield P.M. \& Fisher K. 2005: The evolutionary genetics of egg size plasticity in a butterfly. J. Evol. Biol 18: 281-289.

Summerfield R.J., Roberts E.H., Ellis R.H \& Lawn R.J. 1991: Towards the reliable prediction of time to flowering in 6 annual crops. 1. The development of simple-models for fluctuating field environments. Exp. Agr. 27: 11-31.

Trudgill D.L., Honek A., Li, D. \& van Straalen N.M. 2005: Thermal time - concepts and utility. Ann. Appl. Biol. 146: $1-14$.

VAn Der Have T.M. \& DE Jong G. 1996: Adult size in ectotherms: temperature effects on growth and differentiation. $J$. Theor. Biol. 183: 329-340.

VAn StraAlen N.M. 1994: Adaptive significance of temperature responses in Collembola. Acta Zool. Fen. 195: 135-142.

Van StraAlen N.M. \& Joosse E.N.G. 1985: Temperature responses of egg production and egg development in two species of Collembola. Pedobiologia 28: 265-273.

YADAV J.P. \& Singh B.N. 2005: Evolutionary genetics of Drosophila ananassae III. Effect of temperature on certain fitness traits in two natural populations. J. Therm. Biol. 30: 457-466.

Received March 13, 2006; revised and accepted August 22, 2006 